\title{
ALCALOIDES DAS CASCAS DAS RAÍZES DE Zanthoxylum spp.\#
}

Sandra Virgínia Alves Hohlemwerger, Edijane Matos Sales, Rafael dos Santos Costa e Eudes da Silva Velozo*

Departamento do Medicamento, Faculdade de Farmácia, Universidade Federal da Bahia, Rua Barão de Jeremoabo, s/n, 40170-115

Salvador - BA, Brasil

Maria Lenise da Silva Guedes

Herbário Alexandre Leal Costa, Instituto de Biologia, Universidade Federal da Bahia, Rua Barão de Jeremoabo, s/n, 40170-115

Salvador - BA, Brasil

Recebido em 20/4/12; aceito em 18/9/12; publicado na web em 26/10/12

\begin{abstract}
ALKALOIDS OF ROOT BARKS OF Zanthoxylum spp. In 1959, Gottlieb and Antonaccio published a study reporting the occurrence of lignan sesamin and triterpene lupeol in Zanthoxylum tingoassuiba. In this work we describe the phytochemical study of the root bark of the Z. tingoassuiba which allowed the identification of the lupeol, sesamin, and alkaloids dihydrochelerythrine, chelerythrine, anorttianamide, cis- $\mathrm{N}$-methyl-canadine, predicentine, 2, 3-methylenedioxy-10,11-dimethoxy-tetrahydro protoberberine. The investigation of hexane and methanol extracts of the root bark of Z. rhoifolium and Z. stelligerum also investigated showed the presence of alkaloids dihydrochelerythrine, anorttianamide, cis-N-methyl-canadine, 7,9-dimethoxy-2,3-methylenedioxybenzophen anthridine and angoline. The occurrence of 2,3-methylenedioxy-10,11-dimethoxy-tetrahydro protoberberine is first described in $Z$. tingoassuiba and Z. stelligerum. This is also the first report of the presence of hesperidin and neohesperidin in roots of Z. stelligerum.
\end{abstract}

Keywords: Zanthoxylum tingoassuiba; Z. stelligerum; Z. rhoifolium.

\section{INTRODUÇÃO}

O gênero Zanthoxylum possui mais de 250 espécies, distribuídas mundialmente, especialmente em regiões tropicais e subtropicais. ${ }^{1}$ Este grupo apresenta uma química bastante diversificada, sendo um excelente candidato para as pesquisas, tanto para o isolamento e identificação de marcadores quimiossistemáticos, como os alcaloides benzilisoquinolínicos (Figura 1) característicos das "proto-Rutaceae", ${ }^{2}$ como para a busca por substâncias úteis no desenvolvimento de novos fármacos.

Em 1959, Gottlieb e Antonaccio publicaram estudo relatando a ocorrência da lignana sesamina e do triterpeno lupeol nas cascas do caule de Zanthoxylum tingoassuiba A. St. Hil. ${ }^{3}$ Esta planta era uma das mais de 700 listadas na Farmacopeia Brasileira $1^{a}$ ed. (1926) e sua aplicação na medicina popular como antiespasmódico, analgésico e diurético pode ter sido o mote para este primeiro estudo. ${ }^{4-6} \mathrm{O}$ objetivo deste trabalho foi contribuir com o conhecimento da flora do semiárido, apresentando o resultado do estudo fitoquímico realizado nas cascas das raízes das espécies $Z$. rhoifolium Lam, Z. stelligerum Turez e Z. tingoassuiba A. St. Hil.

O fracionamento e a purificação dos extratos orgânicos das cascas das raízes de $Z$. tingoassuiba possibilitaram a identificação do lupeol (1), da sesamina (2), dos alcaloides benzo[c]fenantridínicos di-hidroqueleritrina (3), queleritrina (4), anortianamida (5) dos<smiles>COc1ccc(C[C@H]2c3cc(O)c(O)cc3CCN2C)cc1O</smiles><smiles>COc1ccc2c(c1O)-c1c(cc3c(c1C)OCO3)C[C@H]2C</smiles><smiles>COc1ccc2cc3[n+](cc2c1OC)CCc1cc2c(cc1-3)OCO2</smiles><smiles>COc1ccc2c(c[n+](C)c3c4cc5c(cc4ccc23)OCO5)c1OC</smiles>

Figura 1. Relações biossintéticas e precursores dos alcaloides presentes nas espécies de Zanthoxylum estudadas

*e-mail: euvelozo@ufba.br

\#Artigo em homenagem ao Prof. Otto R. Gottlieb (31/8/1920-19/6/2011) 
alcaloides protoberberínicos iodeto de $c i s-\mathrm{N}$-metilcanadina (6a) e iodeto de 2,3-metilenodioxi-10,11-dimetoxi-tetra-hidroprotoberberina (8a), além do alcaloide aporfínico iodeto de metil predicentina (7). Nas cascas das raízes de Z. rhoifolium e Z. stelligerum foram identificados os alcaloides benzo[c]fenantridínicos di-hidroqueleritrina (3), anortianamida (5), 7,9-dimetoxi-2,3-metilenodioxi-benzofenantridina (9) e angolina (10); os alcaloides protoberberínicos iodeto de cis-N-metil-canadina (6a) e cloreto de cis-N-metil-canadina (6b). A ocorrência do alcaloide protoberberínico iodeto de 2,3-metilenodioxi-10,11-dimetoxi-tetra-hidroprotoberberina (8a) e do cloreto de 2,3-metilenodioxi-10,11-dimetoxi-tetra-hidroprotoberberina $(\mathbf{8 b})$ são descritas pela primeira vez em Z. tingoassuiba e Z. stelligerum, respectivamente. Este também é o primeiro relato da presença dos flavonoides hesperidina (12) e neo-hesperidina (13) nas raízes de $Z$. stelligerum.

\section{RESULTADOS E DISCUSSÃO}

O extrato metanólico das cascas das raízes de Z. tingoassuiba forneceu um sólido amarelo, identificado como iodeto de metil predicentina (7), Figura 2, através da análise dos dados de espectro de $\mathrm{RMN}{ }^{1} \mathrm{H}\left(300 \mathrm{MHz}, \mathrm{CDCl}_{3}\right)$ e ${ }^{13} \mathrm{C}\left(\mathrm{CDCl}_{3}, 75 \mathrm{MHz}\right)$.<smiles>C=C(C)C1CCC2(C)CC[C@H]3C(CCC4[C@@]3(C)CC[C@@H]3[C@@H](C)[C@@H](O)CC[C@]43C)C12</smiles>

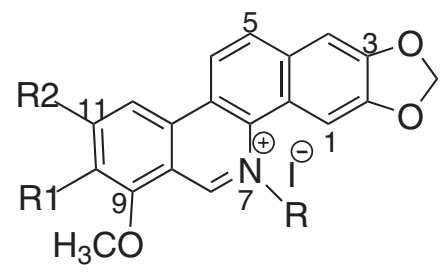

$4 \mathrm{R}=\mathrm{CH} 2 ; \mathrm{R} 1=\mathrm{OCH} 3 ; \mathrm{R} 2=\mathrm{H}$ $9 \mathrm{R}=\mathrm{H} ; \mathrm{R} 1=\mathrm{H} ; \mathrm{R} 2=\mathrm{OCH} 3$<smiles>c1cc2c(cc1C1OCC3C(c4ccc5c(c4)OCO5)OCC13)OCO2</smiles>

2<smiles></smiles>

$3 \mathrm{R}=2 \mathrm{H}$

$10 \mathrm{R}=\mathrm{OCH} 3$<smiles>[R]c1cc2c(c([R])c1[R])C[C@@H]1c3c(cc4c(c3I)OCO4)SC[C@@H]1[N+]2(C)C</smiles>

6a $\mathrm{R}=\mathrm{OCH} 3 ; \mathrm{R} 1=\mathrm{OCH} 3 ; \mathrm{R} 2=\mathrm{H} ; \mathrm{X}=\mathrm{I}^{-}$ 6b $\mathrm{R}=\mathrm{OCH} 3 ; \mathrm{R} 1=\mathrm{OCH} 3 ; \mathrm{R} 2=\mathrm{H} ; \mathrm{X}=\mathrm{Cl}-$ 8a R= H; R1= OCH3; R2; $\mathrm{OCH} 3 ; \mathrm{X}=\mathrm{I}-$ 8b $\mathrm{R}=\mathrm{H} ; \mathrm{R} 1=\mathrm{OCH} 3 ; \mathrm{R} 2 ; \mathrm{OCH} 3 ; \mathrm{X}=\mathrm{Cl}$ -

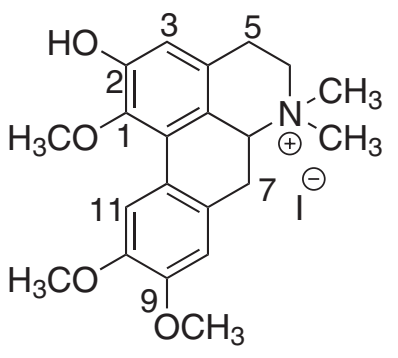

7<smiles>CC(C)=CCOc1c2occc2cc2ccc(=O)oc12</smiles>

11

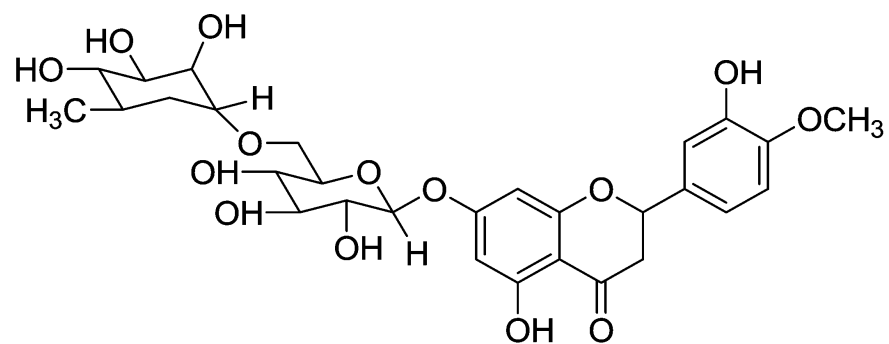

12

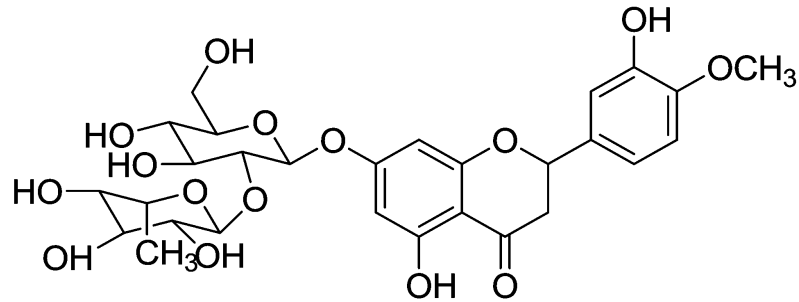

Figura 2. Estruturas isoladas das cascas de raízes de Z. tingoassuiba, Z. rhoifolium e Z. stelligerum 
Este espectro mostra a presença de quatro singletos na região aromática, sendo que um deles $(\delta 6,35)$ tem a intensidade reduzida quando se acrescenta $\mathrm{D}_{2} \mathrm{O}$, indicando a presença de uma hidroxila na estrutura. O espectro revela ainda a presença de três metoxilas em $\delta 3,89 ; 3,93$ e 3,97 e duas $\mathrm{N}$-metilas em $\delta 3,29$ e 3,77. Além disso, os grupos de sinais entre $\delta 4,61-2,88$, com integração total para sete hidrogênios alifáticos são indicativos dos H4a e H4b, H5a e H5b, $\mathrm{H6a}, \mathrm{H7}$ a e H7b presentes nos anéis aporfínicos. O espectro de RMN ${ }^{13} \mathrm{C}$ apresentou doze sinais acima de $\delta 100$ e nove sinais abaixo deste valor, totalizando 21 átomos de carbonos (Tabela 1). Dos nove sinais encontrados, cinco podem ser atribuídos aos carbonos metílicos, sendo três metoxilas e duas $\mathrm{N}$-metilas. O espectro de DEPT $135^{\circ}$ confirma essa atribuição e aponta para a presença de três carbonos metilenos em $\delta 23,9 ; 29,6$ e 61,5 e um carbono metínico em $\delta 70,0$, sendo estes dados compatíveis com a fórmula molecular $\mathrm{C}_{21} \mathrm{H}_{26} \mathrm{NO}_{4}$. A análise dos mapas de contorno HMBC e HMQC (Tabela 1) e comparação com os dados apresentados por Vasquez e colaboradores ${ }^{7}$ foram as ferramentas para o estabelecimento desta estrutura.

Tabela 1. Dados de RMN (300 MHz; $75 \mathrm{MHz} ; \mathrm{CDCl}_{3}$ ) do iodeto metil predicentina (7)

\begin{tabular}{ccccc}
\hline Posição & $\delta \mathrm{C}(\mathbf{7})$ & DEPT (7) & HMQC (7) & HMBC (7) \\
\hline 1 & 147.5 & $\mathrm{C}$ & - & 108,7 \\
2 & 141,9 & $\mathrm{C}$ & - & 108,7 \\
3 & 108,7 & $\mathrm{CH}$ & 6,66 & $147,5-141,9-118,7-23,9$ \\
$3 \mathrm{a}$ & 118,7 & $\mathrm{C}$ & - & 108,7 \\
4 & 23,9 & $\mathrm{CH}_{2}$ & 3,02 & 108,7 \\
5 & 61,5 & $\mathrm{CH}_{2}$ & 4,61 & - \\
$6 \mathrm{a}$ & 70,0 & $\mathrm{CH}$ & 4,46 & - \\
7 & 29,6 & $\mathrm{CH}_{2}$ & 3,42 & 111,6 \\
$7 \mathrm{a}$ & 123,4 & $\mathrm{C}$ & - & 111,6 \\
8 & 111,6 & $\mathrm{CH}$ & 6,99 & $147,8-123,4-29,2$ \\
9 & 147,8 & $\mathrm{C}$ & - & 111,6 \\
10 & 148,3 & $\mathrm{C}$ & - & 112,0 \\
11 & 112,0 & $\mathrm{CH}$ & 7,98 & $148,3-123,9-119,6$ \\
$11 \mathrm{a}$ & 123,9 & $\mathrm{C}$ & - & 112,0 \\
$11 \mathrm{~b}$ & 119,3 & $\mathrm{C}$ & - & - \\
$11 \mathrm{c}$ & 118,7 & $\mathrm{C}$ & - & - \\
$\mathrm{NCH}_{3}$ & 54,7 & - & 3,77 & $70,0-61,5-43,9$ \\
$\mathrm{NCH}_{3}$ & 43,9 & - & 3,29 & $70,0-61,5$ \\
$\mathrm{OCH}_{3}(\mathrm{C} 1)$ & 56,5 & $\mathrm{CH}$ & 3,97 & 147,5 \\
$\mathrm{OCH}_{3}(\mathrm{C} 10)$ & 56,2 & $\mathrm{OCH}_{3}$ & 3,93 & 148,3 \\
$\mathrm{OCH}_{3}(\mathrm{C} 9)$ & 56,0 & $\mathrm{OCH}_{3}$ & 3,89 & 147,8 \\
$\left.\mathrm{OH}_{(\mathrm{C} 2}\right)$ & - & - & 6,35 & - \\
\hline
\end{tabular}

Alíquotas do extrato metanólico das cascas das raízes de Z. tingoassuiba e Z. stelligerum foram tratadas com KI e posteriormente purificadas. Este procedimento permitiu o isolamento dos alcaloides iodeto de 2,3-metilenodioxi-10,11-dimetoxi-tetra-hidroprotoberberina (8a) e iodeto de 2,3-metilenodioxi-10,11-dimetoxi-tetra-hidroprotoberberina $(\mathbf{8 b})$, respectivamente. Essas substâncias foram identificadas através da análise de seus espectros de $\mathrm{RMN}{ }^{1} \mathrm{H} \mathrm{e}{ }^{13} \mathrm{C}$ e comparação com dados da literatura. ${ }^{7}$ Alcaloides protoberberínicos substituídos nas posições 2, 3, 10, 11 como observado na substância (8a), também chamados de pseudoprotoberberínicos, têm ocorrência bastante restrita em relação aos substituídos nos carbonos 2, 3, 9, 10. Giacopello e colaboradores ${ }^{8}$ isolaram o iodeto de 2,3-metilenodioxi-10,11-dimetoxi-tetra-hidroprotoberberina (8a) como intermediário na síntese da fagarina II. Este trabalho descreve pela primeira vez a ocorrência deste metabólito no gênero Zanthoxylum.

O fracionamento e purificação do extrato metanólico da casca das raízes de $Z$. rhoifolium possibilitou a identificação do alcaloide 7,9-dimetoxi-2,3-metilenodioxi-benzofenantridina (pseudonorqueleritrina) (9). A presença da função iminium desblinda o hidrogênio ligado ao $\mathrm{C} 8$. Assim, esta estrutura apresenta, na $\mathrm{RMN}{ }^{1} \mathrm{H}$, um singleto típico $(1 \mathrm{H})$ em $\delta 9,77$. Os demais sinais na região aromática, como dois pares de dubletos, com constantes de acoplamento orto e meta e os dois singletos $\delta 4,14$ e 4,07 (3H), relativos às duas metoxilas são compatíveis com a estrutura de um alcaloide 9,11-dissubstituído. Este raro padrão de substituição em esqueleto benzo[c]fenantridínico tem apenas um registro na literatura. Este trabalho apresentada as atribuições para os carbonos desta estrutura, ampliando as informações apresentadas por Sukari e colaboradores. ${ }^{9}$

\section{PARTE EXPERIMENTAL}

\section{Procedimentos experimentais gerais}

Os espectros de ressonância magnética nuclear ${ }^{1} \mathrm{He}{ }^{13} \mathrm{C}$ (incluídos os experimentos em 2D) foram obtidos nos espectrômetros da marca Varian, Gemini-500 ( ${ }^{1} \mathrm{H}$ : $500 \mathrm{MHz}$ e $\left.{ }^{13} \mathrm{C}: 125 \mathrm{MHz}\right)$; Gemini-300 $\left({ }^{1} \mathrm{H}\right.$ : $300 \mathrm{MHz}$ e ${ }^{13} \mathrm{C}$ : MHz); VNMRSYS-500 $\left({ }^{1} \mathrm{H}: 500 \mathrm{MHz}\right.$ e ${ }^{13} \mathrm{C}: 125$ $\mathrm{MHz})$ e MR-400 ( ${ }^{1} \mathrm{H}: 400 \mathrm{MHz}$ e $\left.{ }^{13} \mathrm{C}: 100 \mathrm{MHz}\right)$, utilizando $\mathrm{CDCl}_{3}$ como solvente e TMS como padrão interno. Nas separações cromatográficas em coluna (CC) foram usadas sílica gel 60 (70-230 mesh) Merck e octadecil funcionalizada (200-400 mesh) Sigma-Aldrich. Nas análises por cromatografia em camada delgada comparativa (CCDC) e preparativa (CCDP) foram utilizadas sílica gel $60 \mathrm{PF}_{254}$ Merck e para a revelação foram utilizados reagente de Dragendorff, vanilina/ácido sulfúrico, vapores de iodo e revelação sob luz UV (254 e 365 nm).

\section{Material vegetal}

As raízes de Z. tingoassuiba A. St. Hil foram coletadas em abril de 2004, em Feira de Santana e as raízes de Z. rhoifolium Lam. e Z. stelligerum Turcz foram coletadas em março de 2003 na Chapada Diamantina - Bahia.

A ratificação taxonômica foi realizada pela Profa MSc. M. L. da S. Guedes curadora do Herbário Alexandre Leal Costa (ALCB), do Instituto de Biologia-UFBA, onde se encontram depositadas as exsicatas sob os números 67894, 60507 e 60508, respectivamente.

\section{Extração e isolamento}

Após secagem e trituração, as cascas das raízes de Z. tingoassuiba (288,0 g), Z. rhoifolium (131,0 g) e Z. stelligerum (195,0 g) foram submetidas à extração contínua por maceração, primeiro com hexano ( 3 x $1 \mathrm{~L})$ e a seguir metanol (3 x $1 \mathrm{~L})$, à temperatura ambiente. O extrato metanólico de Z. tingoassuiba $(56,7 \mathrm{~g})$ foi fracionado em coluna de sílica gel, eluída com (3 x $500 \mathrm{~mL})$ com os seguintes solventes: hexano, diclorometano, clorofórmio, acetato de etila e metanol. Este procedimento forneceu 7 frações (F1-F7). A fração F1 (12,3 g) foi purificada em coluna cromatográfica (CC) em sílica gel, utilizando como eluente mistura de hexano/diclorometano em gradiente de polaridade. Após análise por CCD foram reunidas, conforme a similaridade, em 10 subfrações (G1-G10). A fração G8 (4,5 g) foi submetida a uma CC em sílica gel, utilizando diclorometano/ metanol em gradiente de polaridade. Deste procedimento foram obtidas 21 frações, as quais foram reunidas por similaridade em CCDC, na qual foi isolada a sesamina $(25,3 \mathrm{mg}) \mathbf{2}$. Na fração G9 $(12,4 \mathrm{~g})$ após sucessivas CC em sílica gel, utilizando hexano/diclorometano em gradiente de polaridade, foi possível o isolamento da di-hidroqueleritrina (38,3 mg) 3. A fração F2 (18,1 g) após solubilização em clorofórmio apresentou um precipitado amarelo, que foi retirado da solução por centrifugação; estes cristais foram identificados como queleritrina $(417,0 \mathrm{mg})$ 4. A fração F3 $(4,4 \mathrm{~g})$ foi fracionada em CC em sílica gel tendo como eluente a mistura de hexano e acetato de etila em gradiente, finalizando com metanol. Foram obtidas 8 
frações, de aproximadamente $100 \mathrm{~mL}$ cada, posteriormente reunidas em 5 frações (F3A-F3E). A fração F3A (180,0 mg) foi submetida a uma cromatografia em camada delgada preparativa (CCDP) eluída com hexano e acetato de etila (1:1) obtendo-se um sólido amarelado correspondente à anortianamida $(19,9 \mathrm{mg}) \mathbf{5}$. A purificação da $\mathrm{F} 3 \mathrm{~B}$ (416,0 mg) em CC sílica gel levou à obtenção de um sólido branco identificado como lupeol $(29,1 \mathrm{mg}) \mathbf{1}$. A F3D foi purificada com uma CC sílica gel eluída em clorofórmio e metanol (99:1). Este procedimento, após análise por CCDC, permitiu a obtenção de 5 frações (F3D1-F3D5). Da fração F3D5 foram isolados os alcaloides quaternários de acordo com procedimento descrito por Stermitz e Swineheart. ${ }^{10} \mathrm{~A}$ fração F3D5A foi purificada em CC sílica gel eluída com hexano/clorofórmio, obtendo-se 10 frações após o agrupamento por similaridade. $\mathrm{Na}$ fração 7 foi obtido um precipitado amarelo correspondente ao iodeto de cis-N-metilcanadina $(17,0 \mathrm{mg})$ 6a. A F3D5B foi submetida a uma CC em sílica gel e eluída em clorofórmio e metanol em gradiente de polaridade, obtendo-se 17 frações, as quais depois de agrupadas geraram 6 frações. Da fração 5 foi obtido um precipitado amarelo identificado como iodeto de metil predicentina $(22,0 \mathrm{mg}) 7$. A fração F3D5C foi submetida a sucessivas purificações em CC sílica gel com hexano/clorofórmio em gradiente de polaridade. Estes procedimentos permitiram a obtenção de outro precipitado amarelo claro correspondente ao iodeto de 2,3-metilenodioxi-10,11-dimetoxitetra-hidroprotoberberina $(16,3 \mathrm{mg}) \mathbf{8 a}$.

A metodologia utilizada para a extração e purificação dos extratos hexânicos de Z. rhoifolium e Z. stelligerum foi uma adaptação daquela utilizada por Silva e colaboradores. ${ }^{6}$ Do extrato hexano da casca das raízes de $Z$. rhoifolium (4,2 g) foram isoladas sesamina $(6,0 \mathrm{mg}) \mathbf{2}$, di-hidroqueleritrina $(8,7 \mathrm{mg}) \mathbf{3}$, anortianamida $5(50,0$ $\mathrm{mg}), 7,9$-dimetoxi-2,3-metilenodioxibenzofenantridina (19,6 mg) 9 e angolina $(53,3 \mathrm{mg}) \mathbf{1 0}$. No extrato hexano de Z. stelligerum $(11,1$ g) foram identificados lupeol $(19,6 \mathrm{mg}) \mathbf{1}$, di-hidroqueleritrina $(14,3$ $\mathrm{mg}) \mathbf{3}$, anortianamida $\mathbf{5}(31,5 \mathrm{mg})$, norqueleritrina $(16,0 \mathrm{mg}) \mathbf{9} \mathrm{e}$ imperatorim $(9,6 \mathrm{mg}) \mathbf{1 1}$.

O extrato metanólico de $Z$. stelligerum $(60,4 \mathrm{~g})$ foi tratado com uma solução de ácido acético $3 \%$, ocorrendo a formação de um precipitado, a recristalização deste precipitado em metanol permitiu o isolamento de uma mistura contendo majoritariamente as flavonas hesperidina 12 e neo-hesperidina 13 (3,9 g). A solução aquosa foi submetida a uma partição líquido-líquido com clorofórmio. Este procedimento possibilitou o isolamento de um sólido amarelo, purificado através de $\mathrm{CC}$ de fase reversa (C18), de acordo com metodologia adaptada de Kang e colaboradores. ${ }^{11}$ Este procedimento levou ao isolamento dos alcaloides cloreto de cis-N-metilcanadina $(19,0 \mathrm{mg}) \mathbf{6 b}$ e cloreto de 2,3-metilenodioxi-10,11-dimetoxitetra-hidroprotoberberina $(14,0 \mathrm{mg}) \mathbf{8 b}$.

\section{Iodeto metil predicentina (7)}

$\mathrm{RMN}{ }^{1} \mathrm{H}\left(300 \mathrm{MHz}, \mathrm{CDCl}_{3}\right) \delta_{\mathrm{H}}: 7,98(\mathrm{~s}, 1 \mathrm{H}, \mathrm{H} 11), 6,99(\mathrm{~s}, 1 \mathrm{H}$, H8), 6,66 (s, 1H, H3), 6,35 (s, 1H, OH em H2), 4,61 (m, 1H, H6a), 4,46 (m, 2H, H7), 3,97 (s, 3H, OCH3 em H1), 3,93 (s, 3H, OCH

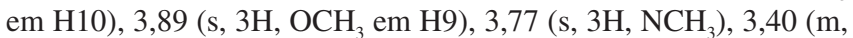
2H, H5), 3,29 (s, 3H, $\left.\mathrm{NCH}_{3}\right), 3,00$ (m, 2H, H4). RMN ${ }^{13} \mathrm{C}\left(\mathrm{CDCl}_{3}\right.$, $75 \mathrm{MHz}) \delta_{\mathrm{C}}$ : Tabela 1.

\section{Iodeto de 2,3-metilenodioxi-10,11-dimetoxitetra-}

hidroprotoberberina $(\mathbf{8 a})$

$\mathrm{RMN}{ }^{1} \mathrm{H}\left(300 \mathrm{MHz}, \mathrm{CDCl}_{3}\right) \delta_{\mathrm{H}}: 6,70(\mathrm{~s}, 1 \mathrm{H}, \mathrm{H} 1), 6,58(\mathrm{~s}, 1 \mathrm{H}$, H4), 3,40-3,20(m, 2H, H5), 4,24 (m, 1H, H6), 3,79 (m, 1H, H6),
5,32 (d, $J=15,3 \mathrm{~Hz}, 1 \mathrm{H}, \mathrm{H} 8_{\text {eq }}$ ), 5,03 (d, $\left.J=15,3 \mathrm{~Hz}, 1 \mathrm{H}, \mathrm{H} 8_{\text {ax }}\right), 6,72$ (s, 1H, H9); 6,77 (s, 1H, H12), 3,40 (dd, $J=18,5 \mathrm{~Hz}$ e $6,3 \mathrm{~Hz}, 1 \mathrm{H}$, $\mathrm{H} 13_{\mathrm{eq}}$ ), 3,07 (dd, $J=18,5$ e 9,6 Hz, $1 \mathrm{H}, \mathrm{H} 13_{\mathrm{ax}}$ ), 4,90 (dd, $J=9,6 \mathrm{e}$ 6,3 Hz, 1H, H14), 3,62 (s,3H, $\mathrm{NCH}_{3}$ ), 3,88 (s, 3H, $\mathrm{OCH}_{3}$ ), 3,85 (s, $\left.3 \mathrm{H}, \mathrm{OCH}_{3}\right), 6,02\left(\mathrm{~s}, 2 \mathrm{H}, \mathrm{OCH}_{2} \mathrm{O}\right) . \mathrm{RMN}^{13} \mathrm{C}\left(\mathrm{CDCl}_{3}, 75 \mathrm{MHz}\right) \delta_{\mathrm{C}}$ : 106,9 (C1), 109,3 (C4), 121,2 (C4a), 24,0 (C5), 51,9 (C6), 66,5 (C8), 119,8 (C8a), 109,7 (C12), 34,8 (C13), 64,0 (C13a), 124,5 (C13b), $50,5\left(\mathrm{NCH}_{3}\right), 56,5\left(\mathrm{OCH}_{3}\right), 56,3\left(\mathrm{OCH}_{3}\right), 102,0\left(\mathrm{OCH}_{2} \mathrm{O}\right)$.

\section{7,9-dimetoxi-2,3-metilenodioxibenzofenantridina (pseudonorqueleritrina) (9)}

RMN ${ }^{1} \mathrm{H}\left(300 \mathrm{MHz}, \mathrm{CDCl}_{3}\right) \delta_{\mathrm{H}}: 8,74(\mathrm{~s}, 1 \mathrm{H}, \mathrm{H} 1), 7,28(\mathrm{~s}, 1 \mathrm{H}$, H4), 7,62 (d, $J=5,4 \mathrm{~Hz}, 1 \mathrm{H}, \mathrm{H} 5$ ), 7,87 (d, $J=5,4 \mathrm{~Hz}, 1 \mathrm{H}, \mathrm{H} 6), 9,77$ (s, $1 \mathrm{H}, \mathrm{H} 8), 8,38$ (d, $J=3,6 \mathrm{~Hz}, 1 \mathrm{H}, \mathrm{H} 10), 8,37$ (d, $J=3,6 \mathrm{~Hz}, 1 \mathrm{H}$, $\mathrm{H} 12), 6,15$ (s, 2H, $\left.\mathrm{OCH}_{2} \mathrm{O}\right), 4,14\left(\mathrm{~s}, 3 \mathrm{H}, \mathrm{OCH}_{3}\right), 4,07$ (s, 3H, $\mathrm{OCH}_{3}$ ). RMN ${ }^{13} \mathrm{C}\left(75 \mathrm{MHz}, \mathrm{CDCl}_{3}\right) \delta_{\mathrm{C}}: 102,0(\mathrm{C} 1), 104,0(\mathrm{C} 4), 119,0$ (C6), 66,5 (C8), 146,0 (C10), 118,0 (C11), 101,0 $\left(\mathrm{OCH}_{2} \mathrm{O}\right), 62,0\left(\mathrm{OCH}_{3}\right)$, $56,0\left(\mathrm{OCH}_{3}\right)$.

\section{CONCLUSÃO}

A ocorrência de alcaloides com origem biossintética na reticulina é um evento comum em espécies do gênero Zanthoxylum. Porém, os alcaloides isolados nas cascas das raízes de Z. tingoassuiba apresentam um padrão de substituição raro, ainda não descrito no gênero. Esta planta, listada na $1^{\text {a }}$ edição da Farmacopeia Brasileira, corre risco de extinção, juntamente com muitos outros vegetais constantes desta obra. O Prof. Gottlieb conhecia a potencialidade da flora nativa e tinha plena consciência da importância do seu estudo há mais de meio século atrás.

\section{AGRADECIMENTOS}

Ao CNPq, à CAPES e FAPESB pelas bolsas e apoios financeiros concedidos.

\section{REFERÊNCIAS}

1. Negi, J. S.; Bisht, V. K.; Bhandari, A. K.; Singh, P.; Sundriyal, R. C.; African Journal of Pure and Applied Chemistry 2011, 5, 412.

2. Waterman, P. G.; Grundon, M. F.; Chemistry and chemical taxonomy of the Rutales, Academic Press: New York, 1983, p. 377.

3. Antonaccio, L. D.; Gottlieb, O. R.; Anais da Associação Brasileira Química 1959, 18,183.

4. Pharmacopoeia dos Estados Unidos do Brasil, $1^{\text {a }}$ ed., Ed. Nacional: São Paulo, 1926.

5. Detoni, C. B.; Cabral-Albuquerque, E. C.; Hohlemwerger, S. V. A.; Sampaio, C.; Barros, T. F.; Velozo, E. S.; J. Microencapsulation 2009, 26,684 .

6. Silva, C. V.; Detoni, C. B.; Guedes, M. L. S.; Velozo, E. S.; Quim. Nova 2008, 25, 2052

7. Vásquez, P. I.; Pérez, E. G.; Slater, E. Y.; Bermúdez, I.; Cassels, B. K.; Bioorg. Med. Chem. 2007, 15, 3368.

8. Giacopello, D.; Deulofeu, V.; Comin, J.; Tetrahedron 1964, 20, 2971.

9. Sukari, M. A.; Salim, W. S. W.; Ibrahim, N. H.; Rahmani, M.; Aimi, N.; Kitajima, M.; Fitoterapia 1999, 70, 197.

10. Stermitz, F. R.; Swineheart, J. A.; Phytochemistry 1980, 19, 1219

11. Kang, J. S.; Long, P. H.; Lim, H. M.; Kim, Y. H.; Blaschke, G.; Arch. Pharmacal Res. 2003, 26, 114. 